\title{
The Role of Ambiguity In Recognition Of Emotional Facial Expressions on the Use of Bodily Contextual Information
}

Belkıs DURMUŞ* Aslan KARAASLAN* Sonia AMADO*

Ege University, Faculty of Letters, Psychology Department, Izmir Turkey
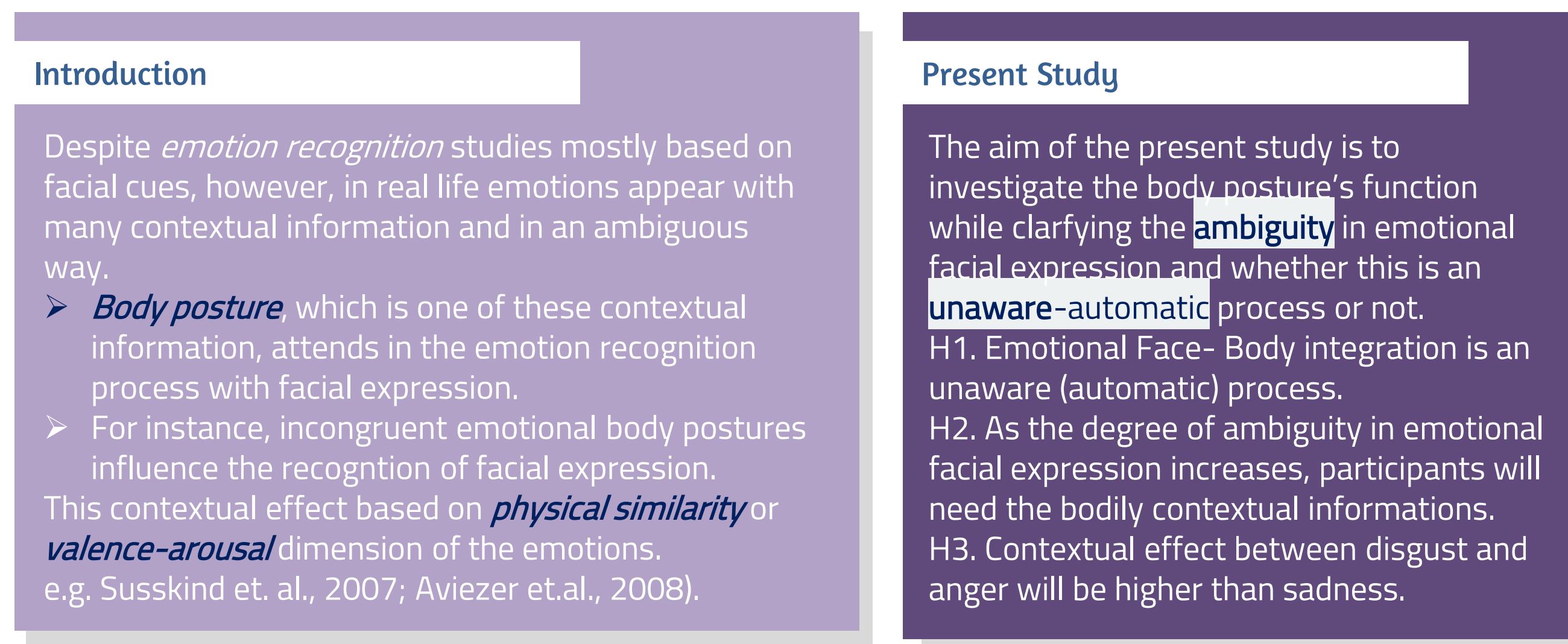

\section{MethodS and Metarials}

77 participants (18 M, 59 F), Mean Age: 20.8

3 emotional faces: angry, disgusted and sad

4 context: angry, disgusted, sad and no-body condition

Face - body pairs were presented briefly (33ms) before face-alone stimulus and participants had to decide the emotional category of the face.

All emotional facial expressions were blended with each other to create ambiguity. * Participants who aware of the body postures were excluded from the analyses.

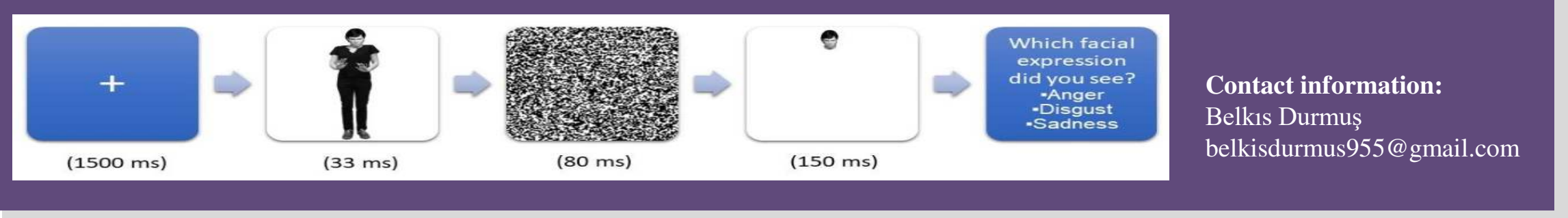

Results

6 (Emotional face - body matches and isolated conditions of emotional facial expressions) $\times 3$ (Anger, Disgust and

Sadness response rates) repeated measures ANOVA was used to test the context effect in terms of ambiguous emotional facial expressions.

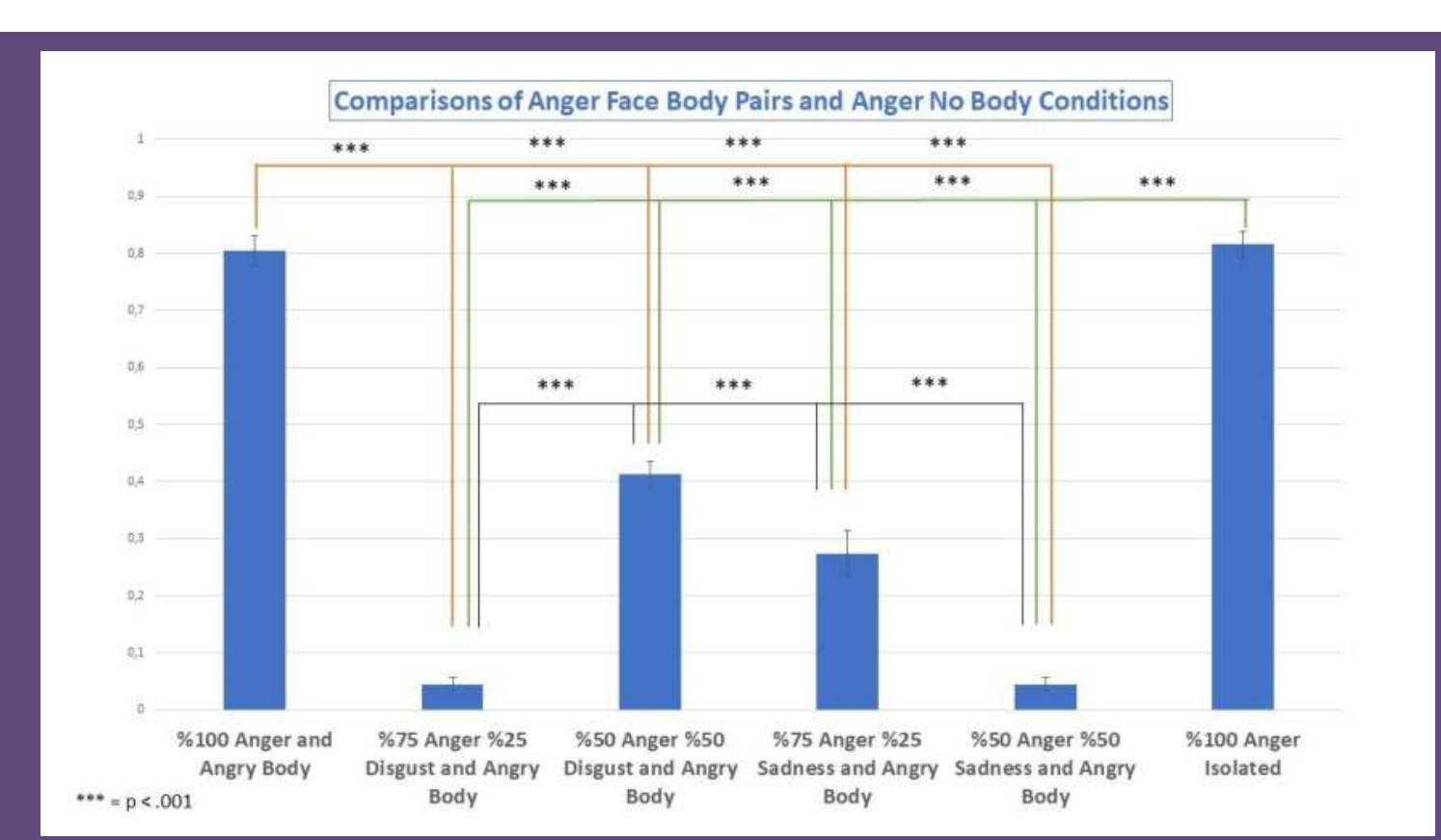

$F(2.855,202.698)=219.899, p<.000$

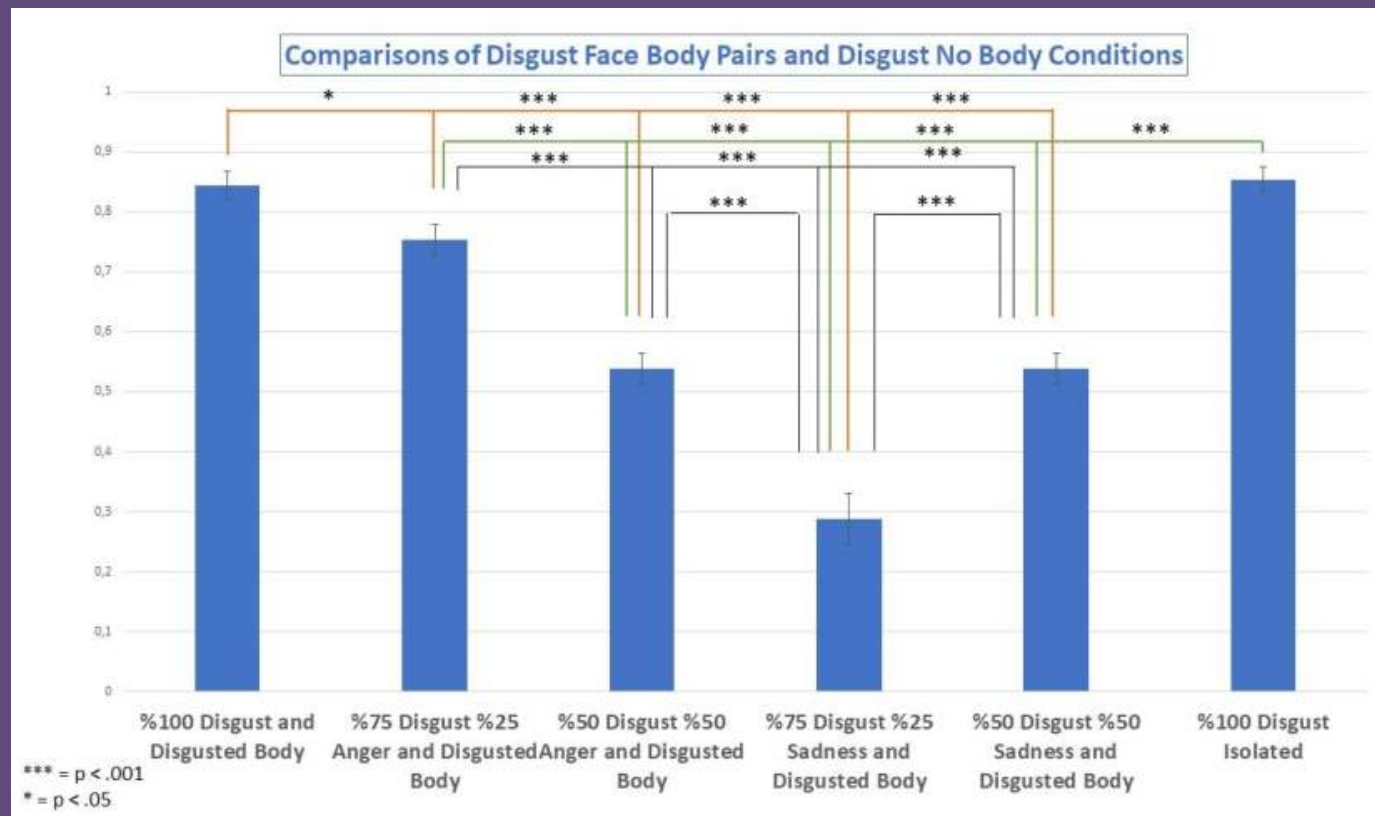

$F(2.743,194.731)=75.994, p<.000$

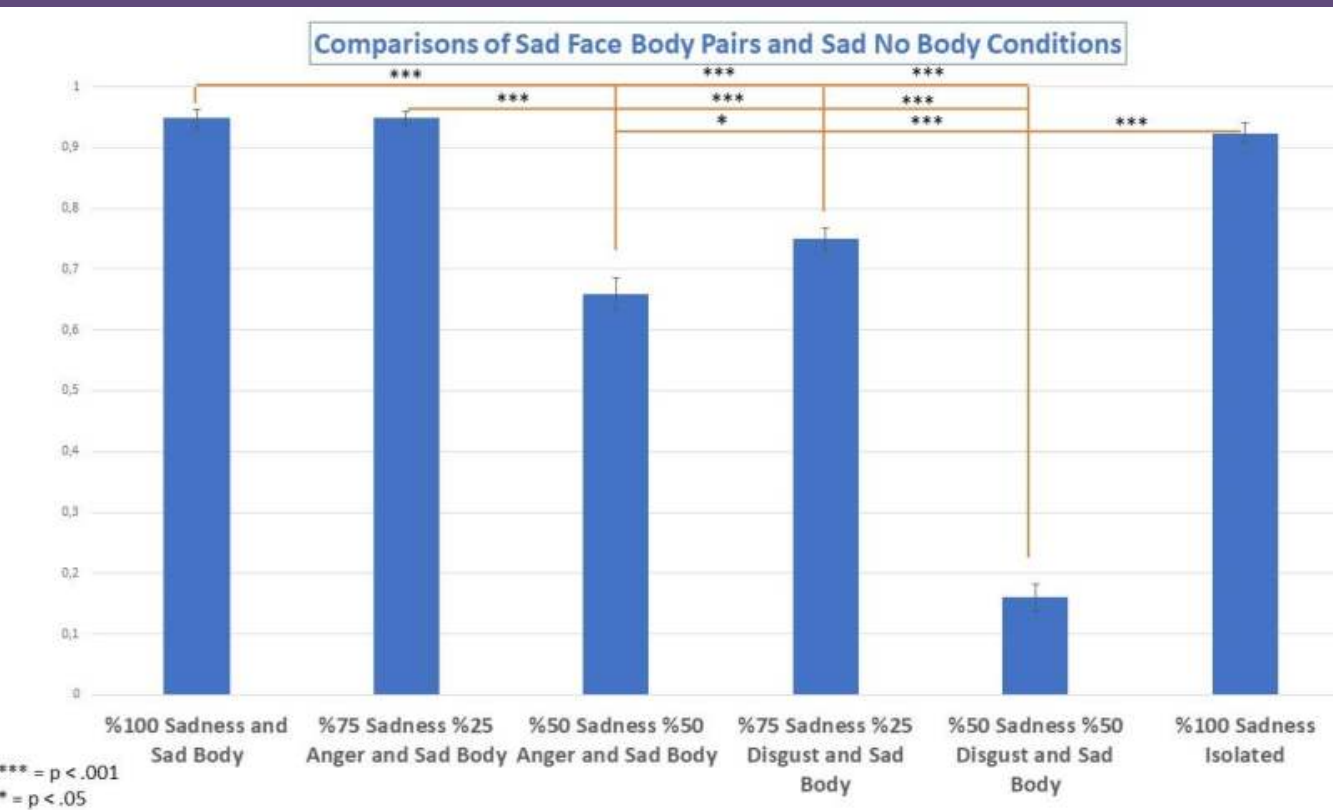

$F(3.702,262.845)=297.516, p<.000$

\section{Conclusion}

$>$ According to the findings, as the ambiguity increases in the angry and disgust face - body matches, the recognition of facial expression is decreased.

$>$ These findings are consistent with past research (e.g. Aviezer et.al., 2008)

However, the condition of high ambiguity (50\% -50\% mixed emotions) in the sadness did not differ from the apparent condition. Yet, sadness, anger and disgust are different in terms of arousal and valence.

Although Aviezer et al. (2008) concluded that the context effect emerges on the basis of physical similaritv (Susskind et_al...2007) this.studvis valuable in that itshows that the effect of context emerges even if the physical similarity is low and that emotional ambiguity of facial expressions is an important determinant of context effect.

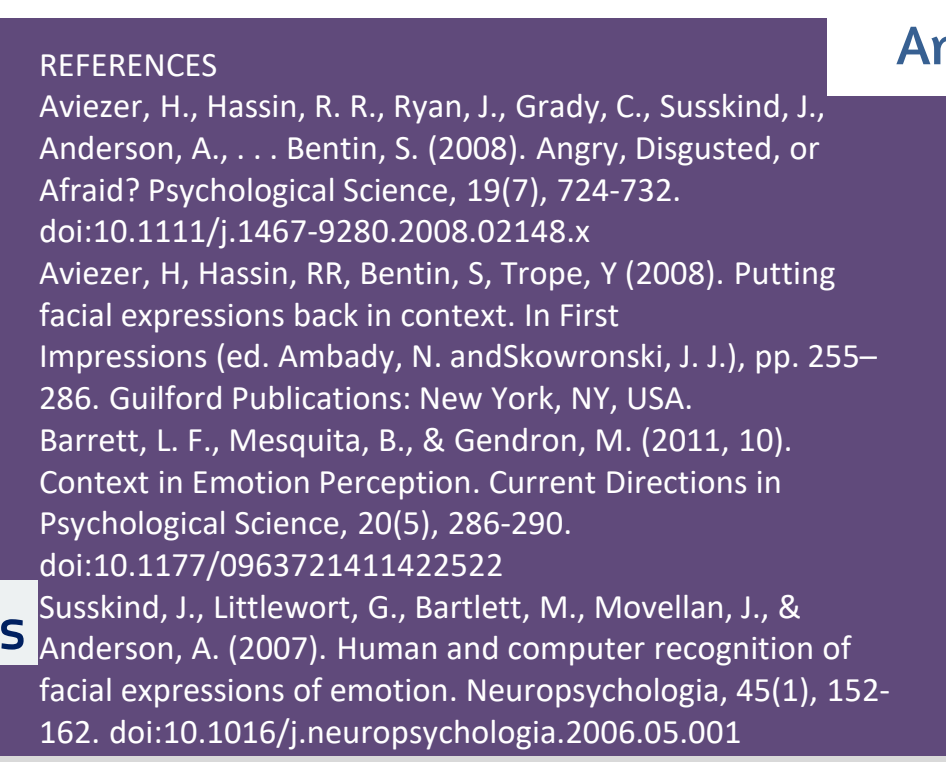

Anger Condition Face - Body Pairs

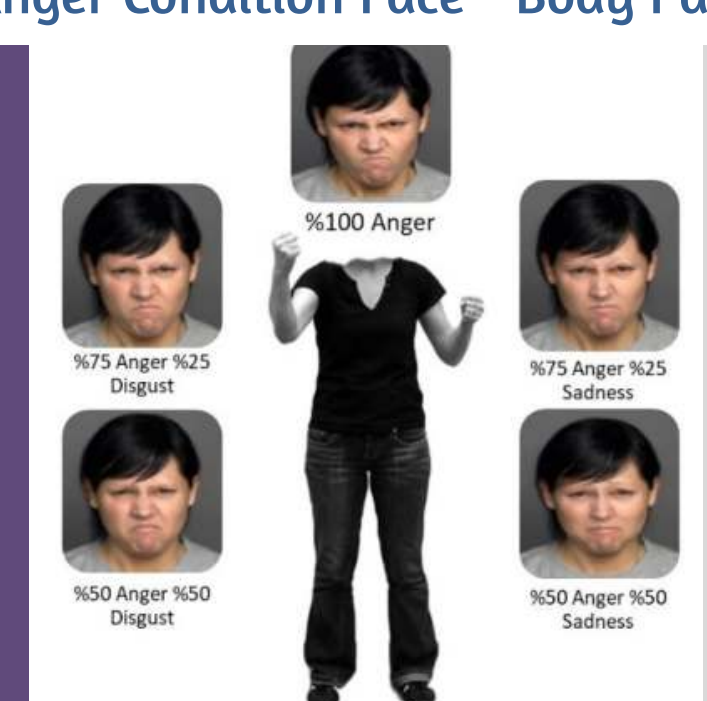

